\title{
Welche Rückenschmerzpatienten profitieren von einer multimodalen Schmerztherapie? Eine komparative Kostenanalyse auf Basis von GKV-Routinedaten
}

\author{
Which Back Pain Patients Benefit From Multidisciplinary Pain Management? \\ A Comparative Cost Analysis Using Claims Data of Statutory Health Insurance
}

Autoren

Institute
S. Behrendt ${ }^{1}$, H. Kulas ${ }^{1}$, U. Marschall ${ }^{2}$, M. Steffens ${ }^{3}$, G. Schiffhorst ${ }^{1}$, H.-H. Bleß

${ }^{1}$ IGES Institut GmbH, Berlin

${ }^{2}$ Barmer GEK, Medizin und Versorgungsforschung, Wuppertal

${ }^{3}$ Grünenthal $\mathrm{GmbH}$, Market Access, Aachen

\section{Schlüsselwörter \\ - Rückenschmerz \\ - Kosten \\ - Responder \\ - Routinedaten \\ - Multimodale Schmerz- therapie}

Key words

- back pain

- responder

- claims data

- multidisciplinary pain management
Bibliografie

DOI http://dx.doi.org/

10.1055/s-0042-110401

Online-Publikation: 21.7.2016

Gesundheitswesen 2016;

78 (Suppl. 1): e120-e127

(c) Georg Thieme Verlag KC

Stuttgart · New York

ISSN 0949-7013

Korrespondenzadresse

Susann Behrendt, M.A.

IGES Institut GmbH

Versorgungsforschung

Friedrichstraße 180

10117 Berlin

Susann.Behrendt@iges.de

\section{License terms}

\section{$(\circledast) \Theta \circledast$}

\section{Zusammenfassung}

$\nabla$

Ziel: Die multimodale Schmerztherapie (MMT) gilt als Goldstandard der Versorgung von Patienten mit chronischen Rückenschmerzen. Die Kosteneffektivität interdisziplinärer Verfahren wurde in diversen Studien belegt. Ziel der vorliegenden Studie besteht darin, jene Patientencharakteristika zu bestimmen, die unter MMT mit günstigen Kostenverläufen assoziiert sind. Angenommen wird, dass ein verbesserter Gesundheitszustand mit einer geringeren Inanspruchnahme von medizinischen Leistungen einhergeht. Zudem sollen jene Rückenschmerzpatienten ohne MMT identifiziert werden, bei denen ein günstiger Kostenverlauf zu erwarten wäre.

Methodik: Auf Basis pseudonymisierter Routinedaten einer überregionalen Krankenkasse wurden Rückenschmerzpatienten mit MMT mit einem geeigneten Grouping-Verfahren identifiziert und je Rückenschmerztyp eine Kontrollgruppe ohne MMT gebildet. Eine komparative Kostenanalyse (indikationsunspezifische Kosten) bestimmte jene MMT-Patienten, bei denen die patientenindividuellen kumulierten Gesamtkosten für beide Postinterventionsjahre geringer waren als die mittleren kumulierten Gesamtkosten der Kontrollgruppe. Eine CART-Analyse identifizierte die statistisch bedeutsamen Merkmalprofile, welche mit diesen günstigen Kostenverläufen assoziiert sind. Die Kontrollpatienten, welche ebenfalls diese Profile aufwiesen, wurden schließlich quantifiziert.

Ergebnis: Es konnten 1252 Patienten mit spezifischen Rückenschmerzen sowie $767 \mathrm{~Pa}$ tienten mit Schmerzen aufgrund von Bandscheibenerkrankungen identifiziert werden, die eine MMT erhielten. Im Vergleich zu den Kontrollgruppen waren die posttherapeutischen indikationsunspezifischen Gesamtkosten der MMT-Patienten höher. Für rund die Hälfte

\section{Abstract \\ $\nabla$}

Aim: Multidisciplinary pain therapy (MPT) is considered as best practice for treating patients with chronical back pain. Several studies showed the cost effectiveness of interdisciplinary treatment. The aim of the present study is to identify patient characteristics that, under condition of MMT, can be associated with favourable cost trajectories after intervention. We assume that an improved health status leads to less health care utilisation. Furthermore, we aim at identifying those patients with back pain without MPT for whom we can expect favourable cost trajectories.

Methods: On the basis of pseudonymised claims data of a nationwide health insurance, we identified MMT back pain patients as well as a control group of back pain patients without MMT. Using comparative cost analysis (costs insensitive to indication), we determined those MMT patients for whom the cumulated total costs per patient for the two postinterventional years were lower than the mean cumulated total costs of the control group. By means of a CART analysis, we identified statistically significant characteristics (profiles) associated with these favourable cost trajectories. Additionally, we quantified control group patients with the same profiles.

Results: The study population comprised 1252 patients with specific back pain and 767 patients with pain due to spinal disc conditions who received MPT. Compared to the control group, total post-therapy costs (insensitive to indication) of MPT patients were higher. For roughly half of MPT patients per pain type, we could identify favourable cost trajectories as per definition. These patients mainly displayed lower levels of pain intensity, no pain-related hospitalisation, and less (mental) co-morbidity in the year preceding the intervention. These profiles concerned to 58$65 \%$ of back pain patients without MPT. 
der MMT-Patienten je Schmerztyp ließen sich per definitionem günstige Kostenverläufe in den Folgejahren feststellen. Je Schmerztyp kennzeichneten die in diesem Sinne profitierenden Patienten insbesondere einen geringeren Schweregrad der Schmerzerkrankung, das Fehlen schmerzbezogener Hospitalisation sowie eine geringere (psychische) Komorbidität im Vorjahr der Intervention. 58-65\% der Rückenschmerzpatienten ohne MMT wiesen diese Profile auf.

Schlussfolgerung: Die entwickelte Methode ermöglicht die Identifikation von Rückenschmerzpatienten, die vermutlich von einer MMT profitieren könnten. Die Studie verweist auf den Bedarf für patientenindividuelles Schmerzmanagement und unterstreicht die Bedeutung, Patienten frühzeitig in multimodale Programme zu integrieren.

\section{Einleitung}

$\nabla$

Chronischer Rückenschmerz zählt zu den häufigsten Erkrankungen in Deutschland [1]. Chronische Rückenschmerzen führen neben beträchtlichen Einschränkungen der Lebensqualität der Betroffenen zu hohen Kosten für das Gesundheitssystem und die Gesellschaft. Die Kosten steigen dabei mit schmerzbezogenem Chronifizierungsstadium und durch Erkrankungskomplikationen wie dem Hinzukommen von neuropathischen Schmerzen oder psychischer Komorbidität [2].

In dem Kontext des gewachsenen Verständnisses der biomedizinischen und psychosozialen Komplexität chronischer Schmerzen wird die Etablierung multimodaler Programme des Schmerzmanagements gefordert. Die 88. Gesundheitsministerkonferenz sprach sich im Juni 2015 explizit für den flächendeckenden Ausbau interdisziplinärer und intersektoraler Versorgungsangebote für Patienten mit chronischen Schmerzen aus. Trotz der Erweiterung von schmerztherapeutischen Strukturen sowohl im ambulanten als auch im stationären Bereich in der jüngsten Vergangenheit erweist sich die Schmerzversorgung in Deutschland nachwievor als defizitär [3].

Die Nationale Versorgungsleitlinie Kreuzschmerz beschreibt die Multimodale Schmerztherapie (MMT) bei Patienten mit chronischen/potenziell chronifizierenden Rückenschmerzen als Goldstandard in der Schmerzversorgung [4]. Die MMT wird definiert als die „gleichzeitige, inhaltlich, zeitlich und in der Vorgehensweise abgestimmte umfassende Behandlung von Patienten mit chronifizierten Schmerzsyndromen (...), in die verschiedene somatische, körperlich übende, psychologisch übende und psychotherapeutische Verfahren nach vorgegebenem Behandlungsplan mit identischem, unter den Therapeuten abgesprochenem Therapieziel eingebunden sind.“ [5].

Dass interdisziplinäre Verfahren im Schmerzmanagement kosteneffektiv sind, belegen Studien im nationalen und internationalen Kontext [6-9]. Angesichts der gestiegenen Anzahl und Diversität multimodaler Programme zur Schmerztherapie gewinnt aktuell die Frage an Bedeutung, welche Schmerzpatienten in der Versorgungsrealität tatsächlich von einer MMT profitieren.

In Deutschland mangelt es jedoch an Studien zur Charakterisierung von Patienten, die auf eine MMT ansprechen. Zudem fehlen Informationen zu Patienten, die keine MMT erhalten, bei denen aufgrund ihrer Charakteristika jedoch ein Therapienutzen zu erwarten wäre. Im Kontext der Planung von Versorgungssteuerungsmaßnahmen stellen die genannten Kenntnisse jedoch essentielle Informationen dar. Ziel der vorliegenden Studie war es, Patientencharakteristika zu identifizieren, die bei Patienten mit
Conclusion: The developed methodology enables identification of back pain patients likely to benefit from MPT. The study points out the need for patient individual pain management and underlines the importance of early-stage integration of patients into multidisciplinary pain management programmes.

MMT mit einer posttherapeutisch günstigen Kostenentwicklung assoziiert sind. Dabei liegt die Annahme zugrunde, dass eine verbesserte Konstitution des Schmerzpatienten zu einer geringeren Ressourceninanspruchnahme führt. Ferner wurde bestimmt, bei welchen Rückenschmerzpatienten, die keine MMT erhielten, ein günstiger Kostenverlauf zu erwarten wäre.

\section{Methodik \\ $\nabla$}

\section{Datengrundlage}

Zur Analyse standen pseudonymisierte Routinedaten einer großen überregionalen gesetzlichen Krankenkasse (BARMER GEK, 8,8 Millionen Versicherte im Jahr 2010) zur Verfügung. Für den Zeitraum 01.01.2009-31.12.2012 lagen Informationen zu Alter und Geschlecht des Versicherten sowie Daten zur stationären und ambulanten Versorgung, zu den ambulanten Arzneimittelverordnungen sowie zu Heil- und Hilfsmitteln vor.

\section{Studienpopulationen}

Die Studienpopulation wurde anhand folgender Kriterien selektiert:

- durchgängig versichert im Gesamtzeitraum 2009-2012,

- Dokumentation von mindestens einem der in 0 Tab. 1 gelisteten MMT-spezifischen Codes im Jahr 2010, jedoch im Jahr vor dieser Intervention (MMT) keinen dieser Codes und

- Alter von mindestens 18 Jahren zu Beginn der Intervention. Da Schmerz als Symptom und Erkrankung bisher weder auf Basis von ICD-Codes noch auf Basis von Arzneimittelverordnungen eindeutig und umfassend klassifiziert wird, entwickelte das IGES Institut im Rahmen des Versorgungsatlas Schmerz ein Verfahren zur Identifikation und Gruppierung von Schmerzpatienten auf Basis von Routinedaten [10,11]. Auf diese Weise konnten 9 unterschiedliche Schmerztypen identifiziert werden ( $\bullet$ Tab. 2). Schmerztypbildende Diagnosen wurden im Jahr vor der Intervention erhoben. Hierbei wurden gesicherte ambulante Diagnosen sowie stationären Hauptentlassungs- und Nebendiagnosen berücksichtigt.

Die Analyse fokussierte auf Patienten mit „spezifischen Rückenschmerzen“ inkl. Osteoporose (Interventionsgruppe sRS) sowie auf Patienten mit „Schmerzen aufgrund von Bandscheibenerkrankungen“ (Interventionsgruppe BS) ${ }^{1}$.

\footnotetext{
${ }^{1}$ Die sogenannten schmerztypbildenden Diagnosen bei sRS gemäß ICD-10 GM sind M43*, M45*, M46*, M48*, M49* sowie M81* und M82*. Bei Schmerztyp BS handelt es sich um die beiden schmerztypbildenden Diagnosen M50* und M51*.
} 
Tab. 1 Identifikation der multimodalen Schmerztherapie auf Basis von Routinedaten der GKV.

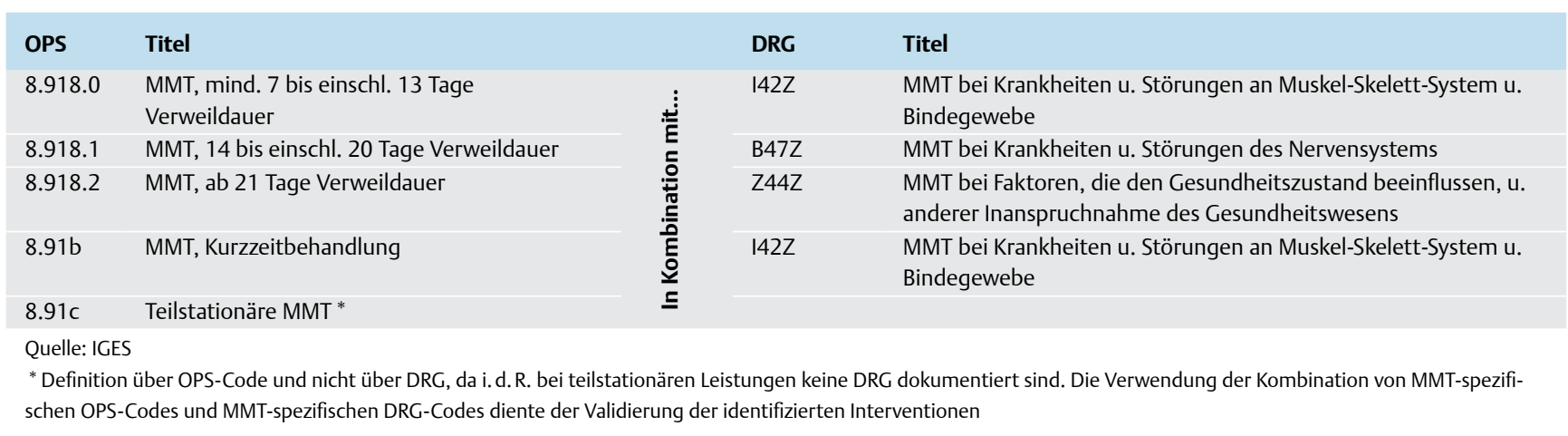

\section{Tab. 2 Schmerztypen gemäß Versorgungsatlas Schmerz*.}

\begin{tabular}{|ll} 
& Schmerztypen \\
\hline 1 & Krebs \\
\hline 2 & (Andere) spezifische Rückenschmerzen \\
\hline 3 & Schmerzen bei Bandscheibenerkrankungen \\
\hline 4 & Arthrose- und Arthritis-bedingte Schmerzen \\
5 & Schmerzen bei traumatischen Frakturen \\
\hline 6 & Schmerzen bei multimorbiden, pflegebedürftigen Patienten \\
7 & Neuropathische Schmerzen \\
\hline 8 & Kopfschmerzen \\
\hline 9 & Nicht spezifische Rückenschmerzen \\
\hline${ }^{*}$ Die Patienten wurden hierarchisch zugeordnet, d. h. Patienten, die einen Schmerz- \\
typ aufwiesen, wurden nachfolgenden Schmerztypen nicht mehr zugeordnet \\
Quelle: IGES nach Versorgungsatlas Schmerz [11]
\end{tabular}

Mittels Propensity Score Matching (PSM) wurde für jede der 2 Interventionsgruppen eine Kontrollgruppe mit Schmerzpatienten ohne MMT im Gesamtzeitraum gebildet, um Strukturgleichheit aller relevanten Kovariaten zu gewährleisten [12]. Potentielle Kontrollpatienten durften dabei im Studienzeitraum von 2009 bis 2012 keine dokumentierte MMT aufweisen und mussten zum Stichtag 01. Juli 2010 mindestens 18 Jahre alt sein. Hierzu wurde ein logistisches Regressionsmodell definiert, in welchem der Erhalt der MMT die abhängige Variable darstellte. Als unabhängige Variablen wurden neben Alter und Geschlecht alle klinisch relevanten Diagnosegruppen nach Clinical Classification System (CCS) [13], Arzneimittelverordnungen auf ATC-2-Level, die schmerztypbezogene ${ }^{2}$ und indikationsunspezifische Inanspruchnahme von medizinischen Leistungen sowie die Gesamtkosten in das Modell aufgenommen. Alle Kovariaten wurden für das Jahr vor der Intervention bzw. bei der Kontrollgruppe für das Jahr vor dem Fixdatum 1. Juli 2010 erhoben. Das PSM basierte auf einem Greedy Algorithmus unter Verwendung des SASMacros von Parsons [12]. Das Matching-Verhältnis betrug 1:1. Hinsichtlich der Kovariaten bestanden für beide Schmerztypen im genannten Zeitraum vor Intervention/Fixdatum keine signifikanten ( $p>0,05, \mathrm{Chi}^{2}$-Test) Unterschiede zwischen Interventions- und Kontrollgruppe.

\section{Identifikation der günstigen Kostenverläufe}

Die Fragestellung, welche Patienten der Interventionsgruppe unter MMT einen günstigen Kostenverlauf zeigen, wurde mittels einer mehrstufigen komparativen Kostenanalyse beantwortet.

\footnotetext{
${ }^{2}$ Schmerztypbezogene Leistungen sind Leistungen, zu denen mindestens eine schmerztypbildende Diagnose dokumentiert wurde.
}

Zugrunde liegt hierbei die Annahme, dass eine verbesserte Konstitution des Schmerzpatienten zu einer geringeren Inanspruchnahme führt und folglich die Kostenentwicklung als Proxy für den Krankheitsverlauf verwendet werden kann. Der Vergleich der Kostenverläufe erfolgte schmerztypbezogen auf Basis der kumulierten indikationsunspezifischen Gesamtkosten (ambulant, stationär, Heil- und Hilfsmittel, Arzneimittel) der Interventions- und Kontrollgruppe für beide Jahre. Ein günstiger Kostenverlauf lag vor, wenn bei MMT-Patienten die kumulierten Gesamtkosten für beide Folgejahre unterhalb der mittleren kumulierten Gesamtkosten der Kontrollgruppe lagen.

Mittels CART-Verfahren (Classification and Regression Tree) wurde für MMT-Patienten mit günstigen Kostenverläufen je Schmerztyp ein Entscheidungsbaum generiert, der aufzeigte, welche Merkmalsprofile mit sinkenden Ressourcenverbräuchen assoziiert sind [14]. Als Prädiktoren im CART dienten demografische und Morbiditätsmerkmale sowie Angaben zu bereits erfolgten Schmerzbehandlungen im Jahr vor MMT.

Abschließend wurde die Anzahl der Patienten der Kontrollgruppe ermittelt, die diese Charakteristika aufwiesen.

\section{Ergebnisse}

\section{Charakteristika der Rückenschmerzpatienten mit und} ohne MMT

Insgesamt 0,04\% $(\mathrm{n}=3177)$ der 8,8 Millionen BARMER GEK-Versicherten (Stichtag 31.12.2010) waren zwischen 2009-2012 durchgängig versichert, wurden im Jahr 2010 multimodal schmerztherapeutisch im Krankenhaus behandelt und waren mindestens 18 Jahre alt. 57,92\% der Patienten waren im Rahmen der MMT 7-13 Tage im Krankenhaus, gefolgt von 31,57\% mit einer Verweildauer von 14 bis 20 Tagen. Eine MMT von mindestens 21 Tagen Dauer sowie die teilstationäre Variante der MMT (Abrechnungsoption erst seit 2009) besaßen im Jahr 2010 zahlenmäßig geringe Bedeutung (2,68 bzw. 7,87\%).

- Tab. 3 charakterisiert die PS gematchten Studienpopulationen je Schmerztyp auf Basis ausgewählter Matching-Parameter.

Die Hochrechnung der Ergebnisse nach Alter und Geschlecht auf die Gesamtpopulation der GKV ergab eine absolute Häufigkeit von 9632 sRS-Patienten mit MMT $(0,01 \%$ der GKV; davon 27,38\% Männer) [15]. 57,72\% dieser Patienten waren mindestens 65 Jahre. Bei den Patienten mit BS handelt es sich um 5901 Versicherte (0,01\% der GKV; davon 34,16\% Männer). Der Anteil an über 64 -Jährigen war mit $24,70 \%$ im Vergleich zum sRS geringer. 
Indikationsunspezifische Versorgungskosten und günstige Kostenverläufe

Die mittleren indikationsunspezifischen Gesamtkosten je Folgejahr sind bei beiden Schmerztypen höher als jene der Kontrollgruppe. Im Jahr vor Intervention/Fixdatum bestanden keine statistisch signifikanten Unterschiede zwischen Interventions- und Kontrollgruppe des jeweiligen Schmerztyps ( $\odot$ Tab. 4). Während die mittleren Jahresgesamtkosten der MMT-Patienten mit sRS im zweiten Jahr 23,53\% höher waren als das Ausgangsniveau (6000 Euro pro Jahr und Patient), sanken die Werte bei der entsprechenden Kontrollgruppe auf minus 3,52\% im zweiten Jahr (Ausgangsniveau: 6039 Euro pro Jahr und Patient). Für die Patienten mit BS ergab sich ein ähnlicher Verlauf ( $\bullet$ Tab. 4). Die stationären Kosten sind bei allen Populationen die kostentreibenden Faktoren. Die Kosten der MMT, die zur Aufnahme in die
Studienpopulation führte, wurden bei den Kostenanalysen nicht berücksichtigt.

Demgegenüber zeigt sich innerhalb der Interventionsgruppe mit sRS bzw. BS für 47,92\% $(\mathrm{n}=600)$ bzw. 48,50\% $(\mathrm{n}=372)$ der Patienten ein günstiger Kostenverlauf. Gemessen an den mittleren Jahresgesamtkosten der Kontrollgruppe weisen diese MMT-Patienten einen kontinuierlichen Kostenrückgang sowie geringere mittlere Kosten als die Kontrollgruppe in jedem Folgejahr auf ( $\bullet$ Tab. 4).

\section{Wer profitiert, wer könnte profitieren - Merkmale potenziell profitierender Patienten}

Für beide Schmerztypen wurden jeweils 3 Profile mittels CART identifiziert, die mit einem günstigen Ressourcenverbrauch assoziiert waren. Diesen Profilen entsprachen 66,17\% $(n=397)$ der

Tab. 3 Charakterisierung der Studienpopulation: Interventions- und Kontrollgruppe je Schmerztyp.

\begin{tabular}{|c|c|c|c|c|c|c|}
\hline & \multicolumn{3}{|c|}{ Spezifische Rückenschmerzen (sRS) } & \multicolumn{3}{|c|}{ Schmerzen durch Bandscheibenerkrankungen (BS) } \\
\hline & MMT (n=1252) & Kontroll (n=1252) & $\mathbf{p}$ & MMT (n=767) & Kontroll ( $n=767)$ & $\mathbf{p}$ \\
\hline \multicolumn{7}{|l|}{ Alter } \\
\hline MW (Jahre) & 65,63 & 65,91 & 0,58 & 55,49 & 56 & 0,41 \\
\hline SD (Jahre) & 12,35 & 13,01 & 0,58 & 12,32 & 12,48 & 0,41 \\
\hline$\geq 65$ Jahre (\%) & 58,63 & 58,07 & 0,78 & 25,81 & 24,77 & 0,64 \\
\hline \multicolumn{7}{|l|}{ Geschlecht } \\
\hline Männer (\%) & 22,12 & 22,60 & 0,77 & 27,38 & 23,99 & 0,13 \\
\hline Frauen (\%) & 77,88 & 77,40 & 0,77 & 72,62 & 76,01 & 0,13 \\
\hline \multicolumn{7}{|l|}{ Morbidität * (\% Patienten) } \\
\hline $\begin{array}{l}\text { Sonst. Diagnose-Codes, u.a. } \\
\text { Schmerzdiagnosen }\left(\text { R52 }{ }^{*}\right)(259)\end{array}$ & 72,84 & 71,65 & 0,50 & 61,93 & 62,58 & 0,79 \\
\hline Hypertonie (98) & 65,26 & 65,58 & 0,87 & 45,76 & 48,11 & 0,36 \\
\hline $\begin{array}{l}\text { Sonst. Knochenerkrankung u. } \\
\text { muskulosk. Deformitäten (212) }\end{array}$ & 63,58 & 63,50 & 0,97 & 57,89 & 56,45 & 0,57 \\
\hline Sonst. Bindegewebsstörungen (211) & 60,86 & 58,23 & 0,18 & 57,76 & 56,45 & 0,61 \\
\hline $\begin{array}{l}\text { Angststörungen, somatof., dissoz. } \\
\text { und Persönlichkeitsstörungen ( } 72 \text { ) }\end{array}$ & 53,83 & 53,04 & 0,69 & 53,19 & 55,54 & 0,36 \\
\hline \multicolumn{7}{|c|}{ Gesamtkosten im Jahr vor MMT/Fixdatum (Euro) } \\
\hline MW & 6233 & 6254 & 0,93 & 4949 & 4808 & 0,62 \\
\hline SD & 6257 & 6479 & 0,93 & 5366 & 5665 & 0,62 \\
\hline
\end{tabular}

Tab. 4 Entwicklung der indikationsspezifischen Jahresgesamtkosten (MW) bei Interventions- und Kontrollgruppe der sRS- bzw. BS-Patienten im Vergleich.

\begin{tabular}{|c|c|c|c|c|c|c|}
\hline & & \multirow{2}{*}{$\begin{array}{l}\text { Jahr vor Einschluss } \\
\text { Jahreskosten (MW) in } \\
\text { Euro (Basisniveau) }\end{array}$} & \multicolumn{2}{|c|}{ 1. Folgejahr } & \multicolumn{2}{|c|}{ 2. Folgejahr } \\
\hline & & & $\begin{array}{l}\text { Jahreskosten } \\
\text { (MW) in Euro }\end{array}$ & $\begin{array}{c}\text { Veränderung zum } \\
\text { Basisniveau (\%) }\end{array}$ & $\begin{array}{l}\text { Jahreskosten } \\
\text { (MW) in Euro }\end{array}$ & $\begin{array}{c}\text { Veränderung zum } \\
\text { Basisniveau (\%) }\end{array}$ \\
\hline \multicolumn{7}{|c|}{ Spezifische Rückenschmerzen (sRS) } \\
\hline \multirow[t]{2}{*}{ gesamt } & MMT (n=1252) & 6000 & 7612 & $+26,86$ & 7412 & $+23,53$ \\
\hline & Kontroll ( $n=1252)$ & 6039 & 5531 & $-8,42$ & 5827 & $-3,52$ \\
\hline \multirow[t]{2}{*}{ stationär } & MMT $(n=1252)$ & 2313 & 3517 & $+52,06$ & 3359 & $+45,23$ \\
\hline & Kontroll $(n=1252)$ & 2573 & 2153 & $-16,32$ & 2272 & $-11,71$ \\
\hline günstig & MMT $(n=600)$ & 3855 & 3299 & $-14,42$ & 2896 & $-24,88$ \\
\hline ungünstig & MMT $(n=652)$ & 7975 & 11581 & $+45,22$ & 11568 & $+45,06$ \\
\hline \multicolumn{7}{|c|}{ Schmerzen durch Bandscheibenerkrankungen (BS) } \\
\hline \multirow[t]{2}{*}{ gesamt } & MMT $(n=767)$ & 4708 & 5902 & $+25,36$ & 6273 & $+33,25$ \\
\hline & Kontroll $(n=767)$ & 4593 & 4403 & $-4,13$ & 4147 & $-9,70$ \\
\hline \multirow[t]{2}{*}{ stationär } & MMT (n=767) & 1605 & 2583 & $+60,97$ & 3015 & $+87,92$ \\
\hline & Kontroll $(n=767)$ & 1802 & 1765 & $-2,07$ & 1446 & $-19,73$ \\
\hline günstig & $\operatorname{MMT}(n=372)$ & 2979 & 2246 & $-24,62$ & 2000 & $-32,87$ \\
\hline ungünstig & MMT $(n=395)$ & 6336 & 9345 & $+47,49$ & 10298 & $+62,53$ \\
\hline
\end{tabular}


MMT-Patienten mit sRS und günstigem Kostenverlauf. Bei den MMT-Patienten mit BS belief sich dieser Anteil auf 72,51\% $(n=266)$. Verordnungen von stark wirksamen Opioiden (WHO-Stufe III) sowie schmerzbezogene Behandlungen vor der Intervention wurden als Proxy für die Schmerzintensität bzw. den Schweregrad verwendet. Sie waren neben Alter und Komorbidität die bedeutsamen Merkmale ( $\odot$ Abb. 1, 2).

$58,07 \%$ der Kontrollpatienten mit sRS und 65,32\% der Kontrollpatienten mit BS wiesen die Profile der MMT-Patienten mit günstigem Kostenverlauf auf.

\section{Diskussion}

Im Rahmen der vorgestellten Studie wurde eine mehrstufige Methode vorgestellt, die eine Identifikation von Patienten ermöglicht, die potenziell von einer MMT profitieren können. Hierzu wurden Profile jener Patienten, die von der MMT profitierten sowie Informationen zu Patienten mit ähnlichen Profilen, die keine MMT erhielten, herangezogen. GKV-Routinedaten geben unzureichend Auskunft über z.B. klinische OutcomeParameter, sodass der Nutzen der MMT über einen Rückgang der Kosten operationalisiert wurde. Für zwei Drittel der Patienten mit sRS sowie drei Viertel der Patienten mit BS und günstigem Kostenverlauf zeigte die CART-Analyse statistisch bedeutsame Profile, d.h. Merkmalsbündel der Patienten, die auf die Therapie ansprachen. Den Großteil der Patienten mit sRS, die auf die MMT ansprachen, kennzeichnet ein Alter von unter 65 Jahren, eine geringe (psychische) Komorbidität, einen geringen Schweregrad der Schmerzerkrankung (operationalisiert v.a. über das Nichtvorliegen von Verordnungen starker Opioide) sowie das Fehlen schmerzbezogener Hospitalisierungen im Vorjahr der Intervention. Beim Schmerztyp sRS verdeutlicht die Analyse jedoch ebenso, dass durchaus auch Patienten im Alter von über 64 Jahren von einer MMT profitieren können, sofern zusätzlich zu den genannten Merkmalen keine Depressionen im Vorjahr der Intervention diagnostiziert wurde und keine Nachsorge-Leistungen (CCS 257, dazu zählen u.a. orthopädische Nachbehandlungen oder Nachbehandlungen nach chirurgischen Eingriffen) vorliegen. Bandscheibenerkrankungen sind ein häufiges Problem von Personen im Erwerbstätigenalter, was letztlich als eine Erklärung dafür dient, dass das Alter bei den MMT-Patienten mit BS keine statistisch bedeutsame Rolle bezüglich eines günstigen Kostenverlaufs spielt.

Vergleichbare Studien auf Basis von Routinedaten der GKV zu den Erfolgsdeterminanten einer MMT finden sich in der deutschsprachigen Literatur nicht. Erkenntnisse zu dieser Fragestellung sind primär Interventionsstudien zu entnehmen, die auf spezifische MMT-Modelle fokussieren, sich auf klinische Daten beziehen und Endpunkte wie Schmerzintensität, Beeinträchtigung und Funktionsfähigkeit der Patienten im Zeitverlauf untersuchen. Unter der Annahme, dass eine verbesserte Konstitution des Schmerzpatienten zu einer kostengünstigen Versorgung im Nachgang der MMT führen kann, zeigen diese Studien - trotz limitierter Vergleichbarkeit zur vorliegenden Analyse - ähnliche Prädiktoren. Bei Heinrich et al. (2011) zählten in der Interventionsstudie zur Effektivität eines tagesklinischen multimodalen Programms für Patienten mit chronischen Rückenschmerzen neben dem Body Mass Index und der Art der Manifestation ebenfalls der Schweregrad des chronischen Schmerzes, aber auch das Ausmaß einer Depression zu den Prädiktoren hinsichtlich der Verbesserung der Funktionskapazität [16]. In weiteren

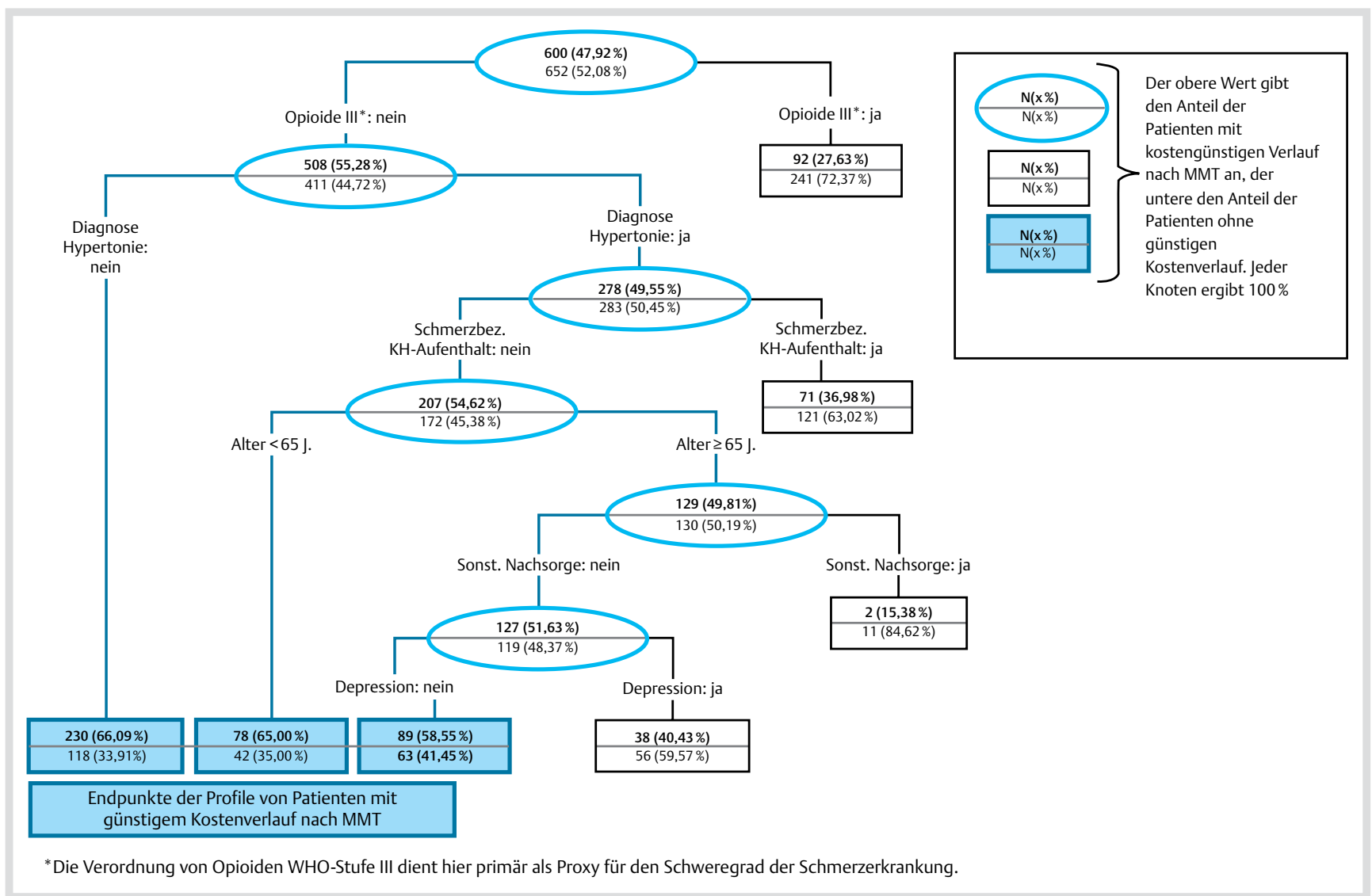

Abb. 1 Profile von sRS-Patienten mit per definitionem günstigen Kostenverlauf nach MMT (Entscheidungsbaum). 


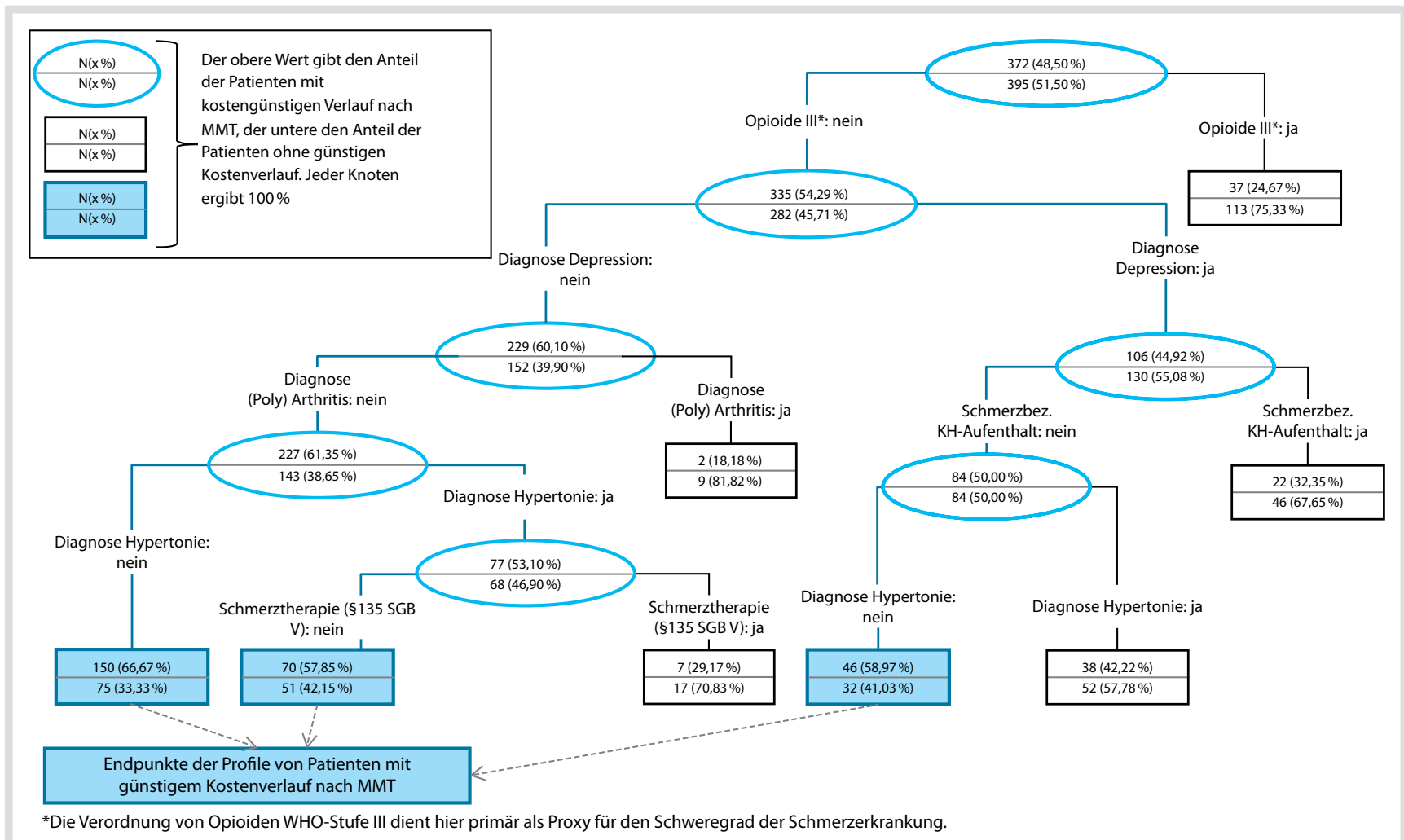

Abb. 2 Profile von BS-Patienten mit per definitionem günstigen Kostenverlauf nach MMT (Entscheidungsbaum).

Studien konnte gezeigt werden, dass eine verbesserte körperliche Funktionsfähigkeit aufgrund der MMT, affektiver Distress, geringere Abwesenheiten vom Arbeitsplatz sowie die Intensität der Inanspruchnahme medizinischer Leistungen vor Initiierung der MMT mit der Schmerzintensität nach der MMT assoziiert sind. [17-19].

Bezüglich der Patienten, die eine MMT erhielten und einen ungünstigen Kostenverlauf aufwiesen, ist zu beachten, dass der ungünstige Kostenverlauf nicht notwendigerweise darauf hinweisen muss, dass diese Patienten nicht von der MMT profitieren können, da unklar bleibt, ob das Ausmaß des ungünstigen Kostenverlauf ohne MMT höher gewesen wäre.

Hinsichtlich der Profile, die im Rahmen der CART-Analyse als Einflussfaktoren auf den Kostenverlauf identifiziert wurden, ist zu vermuten, dass eine hohe Komorbidität und demnach die gleichzeitige Einnahme mehrerer Arzneimittel zu einem ungünstigen Kostenverlauf nach der MMT führen kann [20]. Zum Einen ist durch die zusätzliche Einnahme weiterer Arzneimittel das Risiko für Arzneimittelinterkationen erhöht [21]. Zum Anderen bestehen ggf. Kontraindikationen für einzelne im Rahmen der MMT eingesetzte Arzneimittel, was eine eingeschränkte Effektivität der MMT zur Folge haben kann. Hierfür spricht, dass u. a. ein hohes Alter zu den identifizierten Merkmalsbündeln gehörte. Denkbar ist, dass die ebenfalls identifizierte Hypertension demnach als Proxy-Parameter für eine insgesamt erhöhte Krankheitslast zu interpretieren ist. Im Rahmen der CART-Analyse wurde zudem die Depression als Einflussfaktor auf den Kostenverlauf identifiziert. Hier ist denkbar, dass eine Depression die Adhärenz im Rahmen der MMT einschränkt und somit den Kostenverlauf beeinflussen kann [22]. Zudem führt eine erhöhte Komorbidität dazu, dass verschiedenste Akteure an der Therapie beteiligt sind. Hier deuten Studien darauf hin, dass die Beteili- gung verschiedener Akteure zu teilweise widersprüchlichen Informationen für den Patienten führen kann, was sich wiederum in einer eingeschränkten Adhärenz und einem verminderten Therapieerfolg niederschlagen kann [23].

Die beobachteten Ergebnisse deuten zudem darauf hin, dass Patienten von einem früheren Einsatz der MMT profitieren könnten. In der CART-Analyse zeigte sich, dass ein geringer Einsatz von stark wirksamen Opioiden sowie eine geringe Inanspruchnahme von schmerzbezogenen Krankenhausbehandlungen vor der Initiierung der MMT mit einem günstigen Kostenverlauf nach der MMT assoziiert waren. Auch in anderen Studien konnte andersherum beobachtet werden, dass eine höhere Intensität der Inanspruchnahme von Gesundheitsleistungen einen negativen Einfluss auf z.B. die Schmerzintensität nach Initiierung der MMT hatte und Patienten demnach von einem früheren Einsatz der MMT profitieren könnten [19]. Die Autoren führen jedoch an, dass in Deutschland nach derzeitigem Stand eine MMT erst initiiert werden darf, wenn alle ambulanten Möglichkeiten der Schmerztherapie ausgeschöpft wurden.

Die vorliegende Analyse zeigt außerdem, dass 58\% der sRS-Patienten und 65\% der BS-Patienten ohne MMT (Kontrollgruppen) vermutlich von einer MMT profitieren könnten, da diese Patienten ähnliche Profile aufweisen, wie Patienten, die eine MMT erhielten und einen günstigen Kostenverlauf aufwiesen.

\section{Limitationen}

$\nabla$

Die vorliegende Analyse weist diverse Limitationen auf. Zum einen wurde der Behandlungserfolg in der Interventionsgruppe ausschließlich auf Basis der im Vergleich zur Kontrollgruppe geringeren indikationsunspezifischen Jahreskosten der Folge- 
jahre operationalisiert. Die Kostenentwicklung wurde demnach als Proxy-Parameter für die Entwicklung der Krankheitsschwere herangezogen. Die Analyse der Patienten, die potenziell von der MMT profitieren könnten, basiert auf einer Häufigkeitsauszählung von Kontrollpatienten mit Merkmalsprofilen, die denen von Patienten, die auf die MMT ansprachen, ähnelten. Es erfolgte kein Abgleich der tatsächlichen Kosten dieser Patienten. Bei den vorliegenden Ergebnissen handelt es sich zudem um eine nicht diskontierte Analyse, d.h. es wurde nicht für Menge und Preis kontrolliert.

Es wurde bewusst darauf verzichtet, die Entwicklung der Opioidverbräuche im Nachgang der MMT als Endpunkt einzubeziehen. So können gesteigerte Opioid-Verbräuche ein Proxy für die Zunahme der Erkrankungsintensität sein, ebenso aber auch für eine bessere Integration des Patienten in eine adäquate Versorgung als positiver Effekt der vorangegangenen MMT.

Darüber hinaus sind grundsätzliche Limitationen von GKV-Routinedaten zu nennen. Es handelt sich um Daten zum Zweck der Abrechnung erbrachter medizinischer Leistungen im Kontext der GKV. Klinische Parameter u. a. zur Abschätzung von Schmerzstärken ebenso wie Selbstauskünfte zur Messung der Lebensqualität sind nicht verfügbar.

Eine Hochrechnung der Ergebnisse für die BARMER GEK auf die GKV-Population erfolgte im Hinblick auf die Häufigkeit von MMT-Patienten mit sRS und BS. Einschränkend wirkt hierbei, dass die Studienpopulation im Rahmen des ersten Selektionsschritts ausschließlich die im Zeitraum 2009-2012 durchgängig Versicherten umfasst. Dieser potenzielle Bias ist in der vorliegenden Alters- und Geschlechtsstandardisierung auf die GKV-Population nicht korrigiert, da keine Angaben zu durchgängig Versicherten auf GKV-Ebene vorliegen.

Veränderte Codes zur Dokumentation von Diagnosen bzw. Leistungen führen zu 2 weiteren strukturell bedingten Limitationen: Erst seit 2009 existiert ein OPS-Code für die teilstationäre MMT. Die Häufigkeit der MMT im teilstationären Setting ist daher im Interventionsjahr 2010 sehr niedrig. Die im Rahmen des Versorgungsatlas Schmerz entwickelte Schmerzklassifikation auf Basis von Routinedaten inkludiert nicht die seit 2010 neu etablierten Schmerzdiagnosen (ICD-10) F45.40 Anhaltende somatoforme Schmerzstörung sowie F45.41 Chronische Schmerzstörung mit somatischen und psychischen Faktoren. Unsere Analysen zeigten, dass diese Diagnosen in der Schmerzdiagnostik durchaus relevant sind: Rund 50\% aller MMT-Patienten unabhängig vom Schmerztyp wies mind. einer der beiden Schmerzdiagnosen auf. Hinsichtlich der Schmerzklassifikationen ist darüber hinaus zu beachten, dass sich bei der Zuordnung zu den einzelnen Kategorien Missklassifikationen ergeben können, insbesondere da bei einem Patienten ICD-Codes verschiedener Kategorien dokumentiert sein können. Hinsichtlich der Interpretation der Ergebnisse ergeben sich hieraus jedoch kaum Einschränkungen, da es sich trotz Missklassifikation um einen Patienten mit dokumentierter Schmerzdiagnose handeln würde, dem im Rahmen des PSM ein Kontrollpatient zugeordnet wurde.

\section{Fazit}

Die in der vorliegenden Studie ermittelten Profile von Patienten, die auf die MMT ansprachen, können dazu dienen, Patienten mit chronischen Rückenschmerzen früher in geeignete multimodale Programme zu integrieren. Die Ergebnisse verweisen auf den Bedarf, patientenindividuelle Entscheidungen im Rahmen des Schmerzmanagements zu treffen. Im Hinblick auf die Ausgestaltung der MMT unterstreicht die Studie außerdem die Bedeutung an einer subgruppenspezifischen Ausgestaltung der Angebote, um auf unterschiedliche Chronifizierungsstadien, psychische und somatische Begleiterkrankungen, Schmerzbehandlungshistorien oder auch berufliche Anforderungen des Betroffenen ausreichend Rücksicht zu nehmen.

Mit der entwickelten Methode können potenziell von der MMT profitierende, bisher nicht versorgte Patienten identifiziert, im Rahmen von Pilotprojekten untersucht und bei Bedarf entsprechenden Versorgungssteuerungsmaßnahmen zugeführt werden. Bezüglich der Güte der Prädiktion bedarf es in zukünftigen Studien jedoch einer weiteren Evaluation. Zudem könnten sich weitere Forschungsarbeiten anschließen, in denen geprüft wird, inwieweit die Methodik für andere Versorgungsmaßnahmen und andere Indikationen als jene des chronischen Rückenschmerzes adaptiert werden kann.

\section{Danksagung}

$\nabla$

Die Studie wurde mit finanzieller Unterstützung von der Grünenthal GmbH durchgeführt. Wir danken der Barmer GEK für die Bereitstellung der Daten für diese Studie.

Interessenkonflikt: Die Studie wurde mit finanzieller Unterstützung von der Grünenthal GmbH durchgeführt.

\section{Literatur}

1 Fuchs J, Busch M, Lange C et al. Prevalence and patterns of morbidity among adults in Germany. Results of the German telephone health interview survey German Health Update (GEDA) 2009. Bundesgesundheitsblatt Gesundheitsforschung Gesundheitsschutz 2012; 55 : 576-586

2 Sabatowski R, Kaiser U. Multimodal pain therapy: An established procedure? Schmerz 2012; 26: 644-646

3 Gesundheitsministerkonferenz. 88. Konferenz der Ministerinnen und Minister, Senatorinnen und Senatoren für Gesundheit der Länder am 24. und 25. Juni 2015 in Bad Dürkheim. Ergebnisniederschrift 2015

4 Bundesärztekammer, Kassenärztliche Bundesvereinigung, Arbeitsgemeinschaft der Wissenschaftlichen Medizinischen Fachgesellschaften. Nationale VersorgungsLeitlinie Kreuzschmerz. Langfassung. 2010; Available from: http://www.versorgungsleitlinien.de/themen/kreuz schmerz [Abruf am:17.07.2015]

5 Arnold B, Brinkschmidt T, Casser HR et al. Multimodal pain therapy for treatment of chronic pain syndrome. Consensus paper of the ad hoc commission on multimodal interdisciplinary pain management of the German Pain Society on treatment contents. Schmerz 2014; 28: $459-472$

6 Dietl M, Korczak D. Over-, under- and misuse of pain treatment in Germany. GMS Health Technol Assess 2011; 7: 1-10

7 Nagel B, Korb J. Interdisciplinary treatment. Long-lasting, effective, and cost-effective. Orthopade 2009; 38: 907-908 10-12

8 Kamper SJ, Apeldoorn AT, Chiarotto A et al. Multidisciplinary biopsychosocial rehabilitation for chronic low back pain. Cochrane Database Syst Rev 2014; 9: CD000963 
9 Marschall $U$, L'hoest $H$, Wolik A. Vergleich der Kosteneffektivität von Operation, multimodaler und interventioneller Schmerztherapie bei Rückenschmerzen. BARMER GEK Gesundheitswesen aktuell 2012; 262-285

10 Freytag A, Schiffhorst $G$, Thoma $R$ et al. Identification and grouping of pain patients according to claims data. Schmerz 2010; 24: 12-22

11 IGES Institut GmbH., (Hrsg.). Versorgungsatlas Schmerz. Velbrück Wissenschaft Weilerswist; 2011

12 Parsons LS. Performing a 1: N Case-Control Match on Propensity Score. 2004; Available from: http://www2.sas.com/proceedings/ sugi29/165-29.pdf [Abruf am: 14.10.2015]

13 Healthcare Cost and Utilization Project. Clinical Classifications Software (CCS) for ICD-10-CM/PCS. 2015; Available from: https://www.hcupus.ahrq.gov/toolssoftware/ccs10/ccs10.jsp [Abruf am: 14.10.2015]

14 Breiman L, Friedman J, Stone CJ et al. Classification and Regression Trees. Taylor \& Francis; 1984

15 Bundesministerium für Gesundheit. Gesetzliche Krankenversicherung: Mitglieder, mitversicherte Angehörige und Krankenstand. Jahresdurchschnitt 2010;(Ergebnisse der GKV-Statistik KM1/13) 2011. Available from: http://www.bmg.bund.de/fileadmin/dateien/ Downloads/Statistiken/GKV/Mitglieder__Versicherte/KM1_Jahres durchschnitt_2010.pdf [Abruf am: 14.10.2015]
16 Heinrich M, Hafenbrack K, Michel C et al. Measures of success in treatment of chronic back pain: pain intensity, disability and functional capacity: determinants of treatment success in multimodal day clinic setting. Schmerz 2011; 25: 282-289

17 Hildebrandt J, Pfingsten $M$, Saur $P$ et al. Prediction of success from a multidisciplinary treatment program for chronic low back pain. Spine 1997; 22: 990-1001

18 Michaelson $P$, Sjolander $P$, Johansson $H$. Factors predicting pain reduction in chronic back pain after multimodal treatment. Clin J Pain 2004; 20: 447-454

19 Borys C, Lutz J, Strauss B et al. Effectiveness of a multimodal therapy for patients with chronic low back pain regarding pre-admission healthcare utilization. PloS One 2015; 10: e0143139

20 Pinter G, Likar R, Anditsch $M$ et al. Problems of pain measurement and pain therapy in the elderly. Wien Med Wochenschr 2010; 160: 235-246

21 Syhr KM, Oertel BG, Geisslinger G. Drug interactions in pain therapy. Schmerz 2015; 29: 595-603

22 Graziottin A, Gardner-Nix J, Stumpf $M$ et al. Opioids: how to improve compliance and adherence. 2011; 11: 574-581

23 Chenot JF, Scherer M, Becker A et al. Acceptance and perceived barriers of implementing a guideline for managing low back in general practice. 2008; 3: 7 